\title{
Response monitoring of breast cancer patientsreceiving neoadjuvant chemotherapy using breast MRI - a review of current knowledge
}

\author{
Marc Lobbes ${ }^{1 *}$, Renate Prevos ${ }^{1}$ and Marjolein Smidt ${ }^{2}$ \\ *Correspondence: marc.lobbes@mumc.nl \\ 'Department of Radiology and ${ }^{1}$ Surgery Maastricht University Medical Center, Maastricht, the Netherlands.
}

\begin{abstract}
Background: Breast MRI is the most accurate method for evaluating treatment response in breast cancer patients receiving neoadjuvant chemotherapy (NAC).

Methods: In this review, the current knowledge with respect to response monitoring of breast cancer patients receiving NAC using MRI is presented. Both standard and more advanced approaches are discussed, including potential pitfalls and future developments.

Results: Many parameters have been proposed in the past for early response monitoring of NAC by MRI. Tumour size and volume, and apparent diffusion coefficient values seem most promising and are best validated. However, proper response criteria are still lacking. Breast MRI accuracy for pathologic complete response prediction has moderate sensitivity, but high specificity. Diffusion weighted MR imaging might be able to compensate this shortcoming. However, residual disease assessment after NAC is equally important. Studies have shown that assessment of residual disease by MRI is good, but can be influenced by several factors, such as breast cancer subtype or treatment regimen. Both overestimation and underestimation of residual disease by breast MRI have been reported, and both can have important clinical impact for the individual patient.
\end{abstract}

Conclusion: MRI is the best imaging modality to monitor treatment response in breast cancer patients receiving NAC. However, further research is required to investigate potential confounding factors and its pitfalls.

Keywords: Breast cancer, breast neoplasm, MRI, pathologic complete response, neoadjuvant chemotherapy

\section{Background}

Neoadjuvant chemotherapy (NAC) is defined as the administration of chemotherapy to treat invasive breast cancer before any local treatment, such as surgery. At first, NAC was used for treatment of locally advanced breast cancer. Nowadays, NAC is more and more used in early stages of breast cancer, for example to enable breast conserving therapy (for patients who were originally scheduled for mastectomy), to enable less complicated surgery in cases where skin or pectoral muscle is involved (Figure 1), or to achieve better cosmetic results after surgery. Studies have shown that there is no significant difference in survival or overall disease progression between patients receiving adjuvant or neoadjuvant chemotherapy [1].

The use of NAC enables physicians to assess tumour response in vivo. Patients treated with NAC can demonstrate stable or progressive disease, or remission and even complete response. Complete response is defined as the absence of any residual breast cancer, and is usually assessed by pathological analysis of acquired tissue samples. In this context, the term 'pathological complete response' ( $p C R)$ is frequently used. In general, $\mathrm{pCR}$ is defined as the absence of any residual invasive tumour cells in the original tumour bed. However, the definition of pCR may vary between different grading systems, mainly with respect to the inclusion or exclusion of any residual ductal carcinoma in situ (DCIS) surrounding the culprit region, or with respect to the presence or absence of any axillary lymph node metastases. Pathological complete response after NAC has shown to be a prognostic factor for overall better survival, but also for disease-free survival and recurrencefree survival [2].

Many examinations have been proposed to evaluate response monitoring in breast cancer patients receiving NAC, such as physical examination, mammography, and ultrasound. However, their accuracy was only modest [3]. There is increasing evidence that magnetic resonance imaging (MRI) is an excellent imaging tool to monitor response to NAC, for both early response monitoring and the assessment of residual disease extent. In comparative studies it has been shown that MRI is superior to physical examination, ultrasound, and mammography in response

(C) 2012 Lobbes et al; licensee Herbert Publications Ltd. This is an open access article distributed under the terms of Creative Commons Attribution License (http://creativecommons.org/licenses/by/3.0),This permits unrestricted use, distribution, and reproduction in any medium, provided the original work is properly cited. 

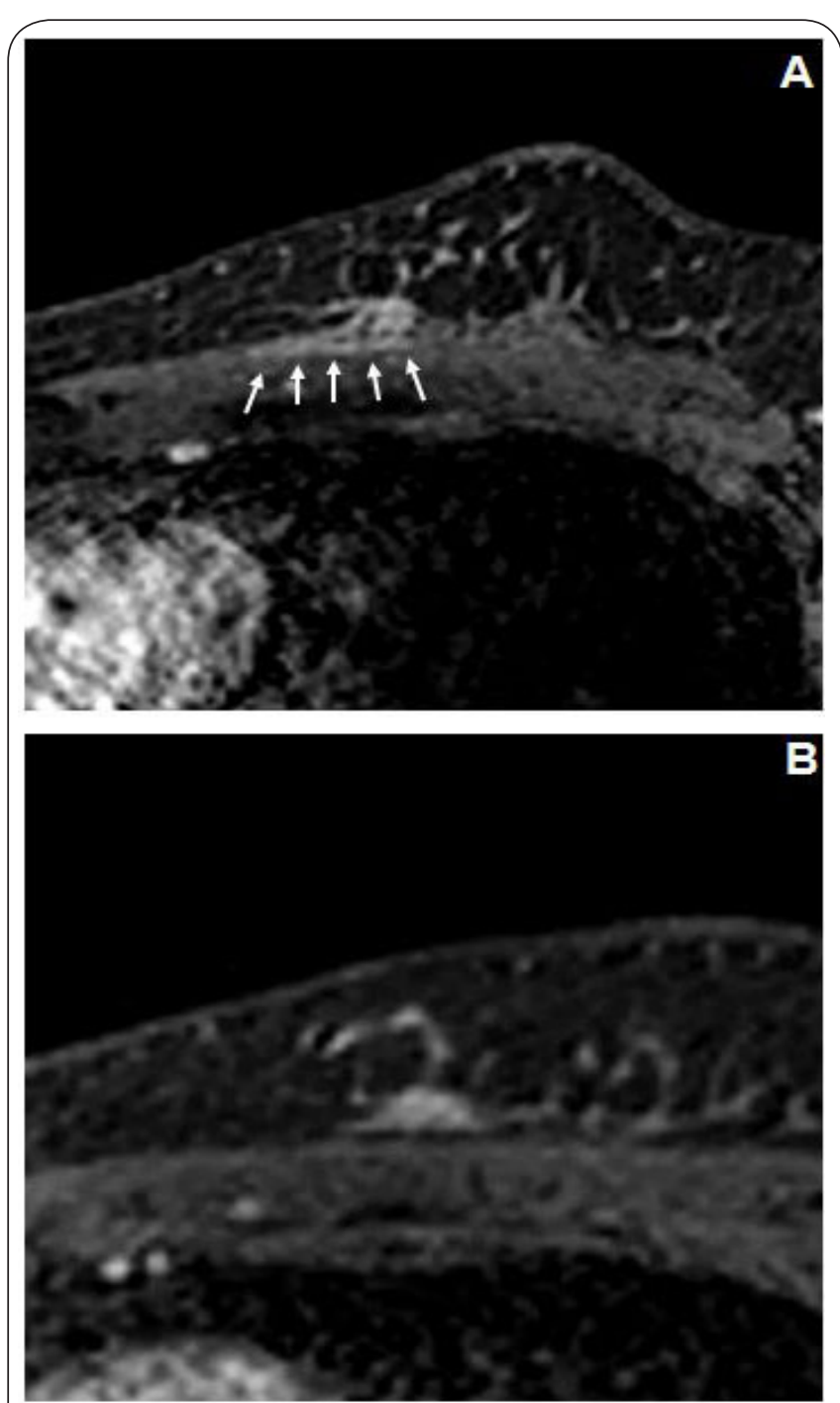

Figure 1. (A) shows a small irregular mass close to the chest wall with increased enhancement of the pectoral muscle ascie medially (total ameter $45 \mathrm{~mm}$ ). After neoadjuvantchemtherapy (B), mass size has not changed significantly, but the enhancement of muscle fascie has disappeared and a fatty line has occured between tumor tissue and chest wall, thus enabling breast conserving therapy in a patient who would otherwise undergo mastectomy.

evaluation during NAC [4]. It is able to monitor complete response, partial response and poor response (Figure 2).

Because NAC allows the physicians to monitor disease development in vivo, there is a need for a (preferably noninvasive) modality that is able to assess tumour response during treatment. There is a need for a modality that can assess response early in treatment (i.e. after only several cycles of chemotherapy), but also for a modality that can accurately detect any residual disease left after therapy. This review focuses on the role of magnetic resonance imaging with respect to response monitoring in breast cancer patients receiving NAC. We attempted to provide
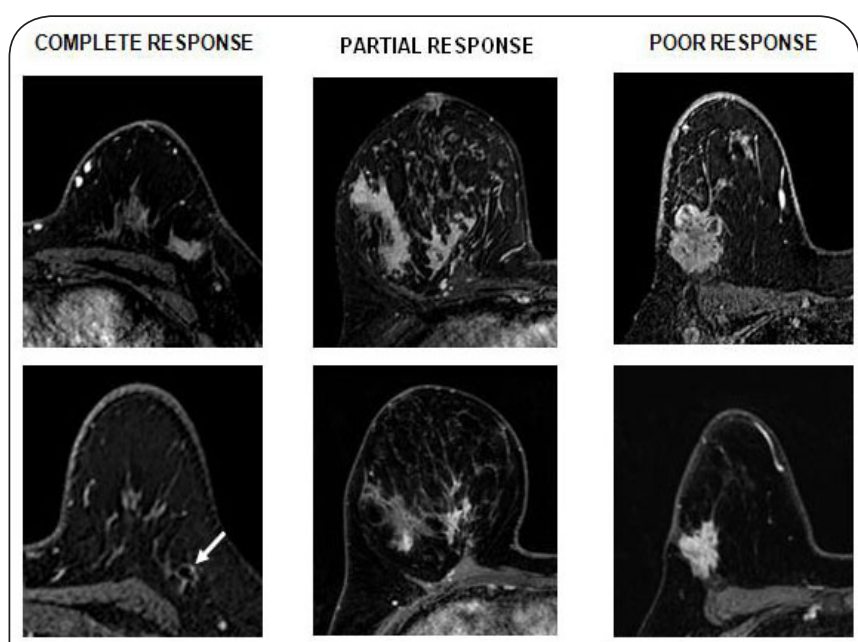

Figure 2. All images are contrast-enhanced, T1-weighted MR images. Top row shows baseline MR examinations, whereas the bottom row shows MR examinations after completion of neoadjuvant chemotherapy. Left column shows an example of complete response, in which the tumour disappeared completely and only the marker clip artifact (arrow) is still visible (Miller and Payne grade 5); middle column shows partial response, with some enhancing residual disease left (Miller and Payne grade 3); right column shows poor response (Miller and Payne grade 2).

the reader with an overview of current knowledge and developments, and explore the lacks in knowledge that still remain. Different techniques for assessing early response in NAC are discussed, as well as the accuracy of MRI to assess residual disease extent and $\mathrm{PCR}$. These issues are illustrated by several examples from everyday clinical practice.

\section{Early response monitoring}

Early response monitoring is considered to be the monitoring of disease changes after the first cycle(s) of NAC treatment. In other words, it is the assessment of disease changes before the completion of the entire course of NAC.

With respect to early response monitoring, many studies have been published, which have been summarized in a recent meta-analysis by our study group, and which included a total of 15 studies until January 2012. In this review, a total number of 31 potential MR parameters were described [5]. The MRI techniques that were most frequently used were dynamic, contrast-enhanced MRI (DCE-MRI), diffusion weighted-imaging (DWI), and MR spectroscopy (MRS).

\section{Dynamic, contrast-enhanced MRI}

DCE-MRI is based on the fact that breast cancers possess an increased microvasculature, which is often 'immature' and leaky, resulting in a leakage of contrast agent through the vessel walls into the interstitium. As a result, breast tumours show enhancement after contrast administration. In DCEMRI, two separate approaches can be identified for early response monitoring in NAC: pharmacokinetic modeling using 'ultrafast' DCE-MRI and 'standard' contrast-enhanced 
Lobbes et al. Journal of Cancer Therapeutics \& Research 2012,

http://www.hoajonline.com/journals/pdf/2049-7962-1-34.pdf

doi: $10.7243 / 2049-7962-1-34$

breast MRI. These techniques can either be performed alone, or combined in a dedicated sequence protocol.

For the assessment of different pharmacokinetic parameters, it is mandatory that a MR sequence is used with a very small temporal resolution, usually in the order of several seconds. As a result, spatial resolution is often poor, preventing adequate assessment of lesion morphology. With respect to early response monitoring in NAC, many parameters of pharmacokinetic modeling have been proposed, such as $\mathrm{K}^{\text {trans }}, \mathrm{K}_{\mathrm{ep}}$ and $\mathrm{V}_{\mathrm{e}}$. However, there are several characteristics of pharmacokinetic modeling (which are general and not specific for the NAC setting) which hamper the widespread use of this approach. For example, the measurement of the arterial input function (AIF) can be difficult. The AIF is the concentrationtime curve of the artery supplying the vascular bed of interest, which in breast MRI is most commonly the internal mammary artery or aorta. For proper measurement of the AlF, a high temporal resolution is essential to capture the inflow and passage of contrast in the blood stream. However, the artery that is used is often located at an important distance from the breast tumour. As a result, temporal resolution is reduced in order to fit the artery within the field-of-view. This leads to an inaccurate assessment of the AIF and pharmacokinetic parameter outcomes. The parameters could also be influenced by the dose and type of the contrast agent used, and by the method by which the contrast agent is administered. Finally, the MR sequence composition can be of influence on these parameters as well $[6,7]$. As a result, many variables can be introduced in pharmacokinetic modeling, which make it difficult to compare different studies. This is probably the most important reason why the use of pharmacokinetic modeling has not been widely introduced in everyday clinical practice after all these years.

In their meta-analysis, Prevos et al., observed both significant and non-significant changes in both absolute and relative values of $\mathrm{K}^{\text {trans }}, \mathrm{K}_{\mathrm{ep}}$, and $\mathrm{V}_{\mathrm{e}}$ between responders and non-responders. In order to be clinically applicable, it would be interesting to learn what the area under the ROC curve (AUC) would be for these parameters. Based on the AUC, a cut off value for absolute or relative changes in the different parameters could be suggested in which the diagnostic accuracy would be optimal. Although several studies presented AUC values for $\mathrm{K}^{\text {trans }}$ and $\mathrm{K}_{\mathrm{ep}}$, this was not available for Ve. For $K^{\text {trans }}$ and $K_{e^{\prime}}$ this AUC ranged from 0.6-0.9 [8-11]. Disappointingly, only a single study suggested absolute and relative cut off values of $0.39 \mathrm{~min}^{-1}$ and $85 \%$, respectively [12]. Currently, the aforementioned limitations of this technique and the scant scientific evidence that is currently available prevent widespread application in everyday clinical practice.

As an alternative, 'standard' DCE-MRI might be used for early response monitoring. In this approach, lesion morphology and extent is assessed by evaluating contrast-enhanced, T1weighted images using a dedicated breast coil and minimum field strength of 1.5 Tesla. Additional information can be extracted by analyzing the signal-intensity time curves in
BEFORE TREATMENT

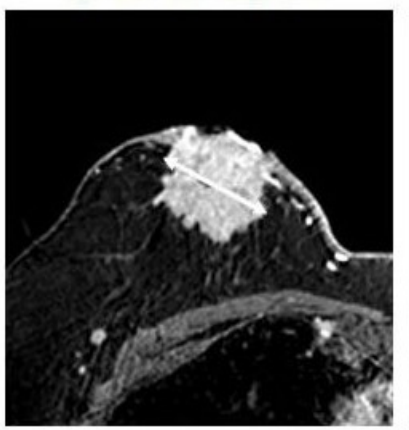

AFTER TREATMENT

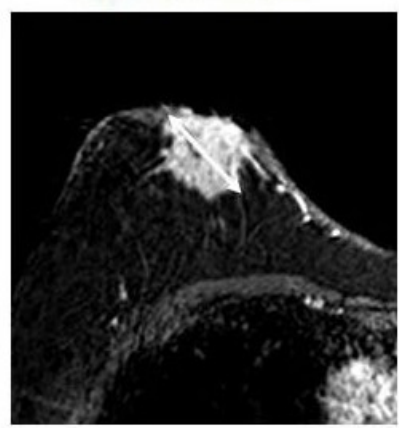

Figure 3. A large, irregular mass with skin involvement is visible in the right breast. Maximum tumour diameter before treatment was $38 \mathrm{~mm}$ (arrow). Maximum tumour diameter after treatment was 32 $\mathrm{mm}$ (arrow). This is a diameter reduction of $16 \%$, corresponding to 'stable disease' according to RECIST and WHO criteria.

Histopathologic analysis showed poor tumour regression, i.e. Miller and Payne grade 2.

the contrast-enhanced dynamic series [13].

In a study of 216 breast cancer patients, Hylton et al., evaluated changes in tumour volume and diameter after the first cycles of treatment with NAC. They observed that early in treatment, maximum tumour diameter and tumour volume were the best parameters to predict PCR (AUC of 0.64 and 0.70 , respectively) [4]. In our systematic review, we found four studies presenting AUC values, which were slightly higher and ranged from 0.73 to 0.9 [5]. Similar to the pharmacokinetic parameters, the majority of the studies did not suggest useful cut off values at which diagnostic accuracy would be optimal. Only Loo et al., suggested a cut off value of a diameter reduction of $25 \%$ between MRI examinations at baseline and at first follow-up [14].

Although evidence is still rather limited, the measurement of tumour volume and diameter seems promising. As an additional advantage over pharmacokinetic modeling parameters, clinical breast MRI has been standardized to a large extent over the past years. With respect to clinical application, measurement of tumour diameter is perhaps the simplest method of (early) response monitoring. Nonetheless, there are several important limitations to these measurements.

With respect to maximum tumour diameter measurement, the two most commonly used guidelines to assess treatment response are the guidelines of the World Health Organization (WHO) and the Response Evaluation Criteria for Solid Tumours (RECIST). In the WHO guidelines, four response categories can be identified: (1) complete response $(\mathrm{CR})$, in which no more enhancing tumour is visible; (2) partial response (PR), which is a reduction in diameter of $\geq 50 \%$, (3) progressive disease (PD), which is an increase in tumour diameter of $\geq 25 \%$ and which rarely occurs under NAC; and (4) no changes, which is the level between PD and PR $[15,16]$. The RECIST criteria also divide response into four categories: $C R$ is defined as the 


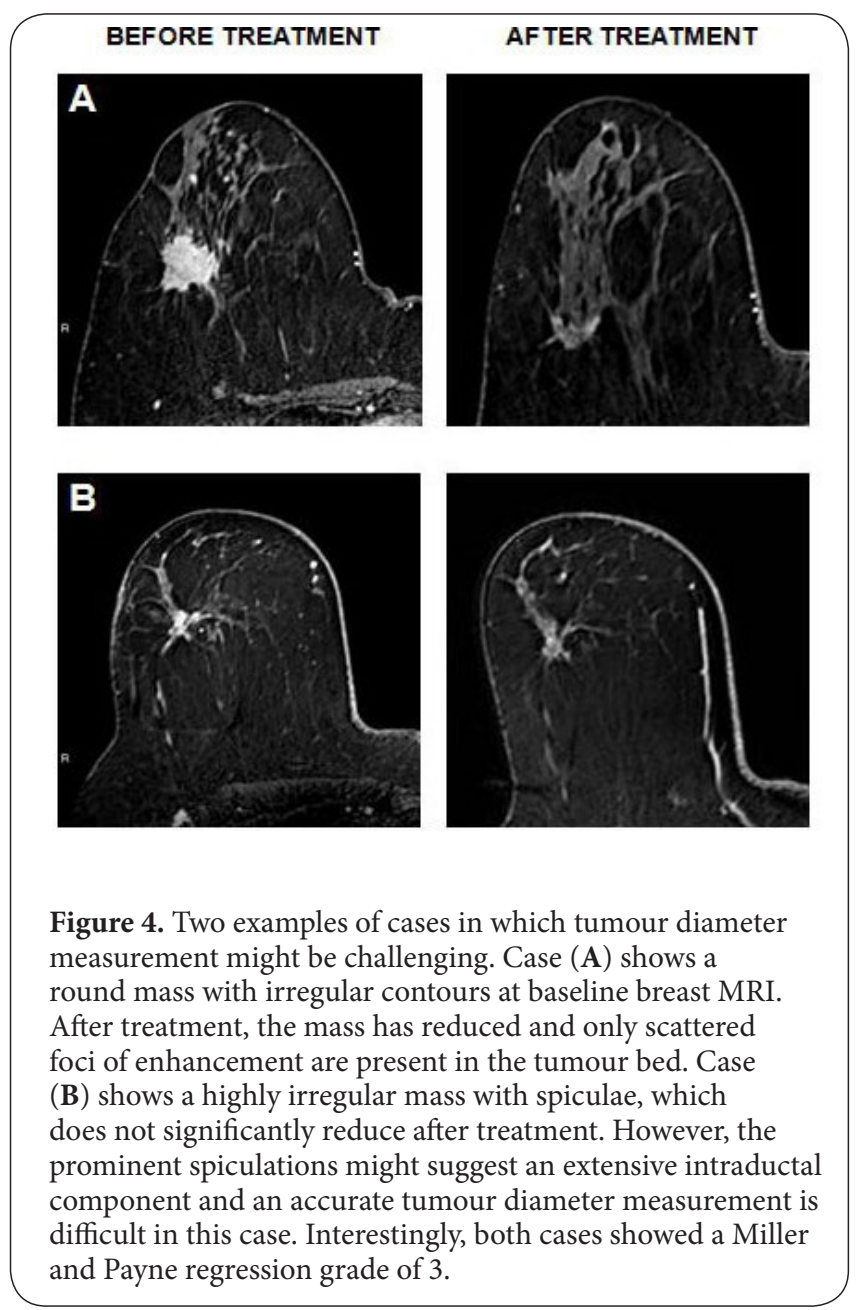

absence of enhancing tumour; PR as a decrease in tumour diameter $\geq 30 \%$; PD as an increase in tumour diameter $\geq 20 \%$; stable disease is considered to be the level between PD and PR (Figure 3) $[16,17]$. However, accurate measurement of tumour diameter can be challenging in cases which show fragmentation of tumour during NAC, or in cases which show a mass with surrounding areas of so-called non-mass like enhancement (which can be associated with a more extensive intraductal component (Figure 4) [18].

To cope with these difficulties, some studies have suggested the use of tumour volume measurements as treatment response parameter $[4,5,16]$. However, there are no well-validated response criteria available for tumour volume measurements. Some studies used the surrogate parameter of tumour volume measurements that was provided in the RECIST criteria appendix: PR was defined as a volume reduction of $\geq 65 \%$, whereas PD was defined as an increase in tumour volume of $\geq 20 \%$ [19-21]. For our study population of interest (i.e. breast cancer patients receiving $N A C$ ), these response criteria are not validated in large, multicenter studies and thus should be used with this limitation in mind. Until today, the lack of proper response criteria and the variation in methods to determine tumour volumes [16] have prevented widespread use of tumour volume assessment as parameter for treatment response in breast cancer patients receiving NAC.

\section{Diffusion-weighted imaging}

In DWI, the random Brownian movement of water molecules is evaluated using MRI. The free (or restricted) movement of these molecules can be quantified by calculating the apparent diffusion coefficient (ADC). In breast cancer, dense packing of cells results in a restricted movement of water molecules. As a result, the ADC value decreases in DWI. During NAC, cell density of the tumour generally decreases, improving water molecule movement within the tumour. As a consequence, the ADC increases during NAC if a proper response is expected. If $A D C$ values remains stable or even decreases further, it would be suggestive for stable or progressive disease under NAC. Thus, measurement of ADC might be a suitable parameter for evaluating treatment response.

In the study by Sharma et al., tumour volume, diameter and ADC value were assessed at each MRI examination. They showed that the percentage change in ADC after the first cycle of treatment was statistically significant between responders and non-responders, when compared with tumour volume and diameter. After three cycles of NAC, sensitivity and specificity of the ADC-value for distinguishing responders and non-responders were $68 \%$ and $100 \%$, respectively [22]. a study by Fangberget et al., an ADC cut off value of $1.42 \times 10^{-3} \mathrm{~mm}^{2 / \mathrm{s}}$ (at $b=750 \mathrm{~s} / \mathrm{mm}^{2}$ ) was suggested as optimal value after four cycles of chemotherapy [23]. This yielded a sensitivity and specificity to distinguish responders and non-responders of $88 \%$ and $80 \%$, respectively. A significant change in $A D C$ in responders after several cycles of NAC was also observed in a study by Li et al., This change could not be observed in non-responders. However, they did not suggest any kind of cut off value at which diagnostic accuracy would be optimal [24].

In summary, several studies have shown the potential of DWI to predict early response in breast cancer patients receiving NAC. However, the number of studies is still limited, and the size of the various study populations is rather small. In addition, the use of DWI has several limitations. For example, DWI has limited spatial resolution and as a result, small tumour foci might be overlooked. In addition, breast cancer subtypes that do not form masses might be misinterpreted (i.e. ductal carcinoma in situ or invasive lobular carcinoma). Furthermore, DWI is a technique that is highly sensitive to motion artifacts caused by (in)voluntary patient movement, resulting in inaccurate $A D C$ values due to imaging mismatch. Finally, DWI sequences can vary between vendors and institutions, especially with respect to the select $b$-values in this technique, which influence the $A D C$-value and make it difficult to compare study results between different groups. 
Lobbes et al. Journal of Cancer Therapeutics \& Research 2012,

http://www.hoajonline.com/journals/pdf/2049-7962-1-34.pdf

doi: $10.7243 / 2049-7962-1-34$

\section{Magnetic resonance spectroscopy}

In MRS, the molecular composition of a tissue of interest is analyzed by looking at the spectrum of resonances produced by the MR signal. This spectral 'signature' is used to diagnose certain metabolic disorders that are associated with tumour cells. The majority of the MR signal arises from fat and water, but small magnetic field differences can be utilized to identify additional metabolites that are present in much smaller concentrations. Relative to normal breast tissue, breast cancers have increased levels of choline-containing compounds (such as phosphocholine, glycerophosphocholine, and free choline). However, these metabolites are present in extremely small concentrations, and cannot be identified separately by MRS. Therefore, the total choline peak (tCho, at $3.2 \mathrm{ppm}$ ) is usually presented [16].In a study by Jagannathan et al., 67 patients with breast cancer and 16 healthy controls were evaluated. Using MRS, they showed that sensitivity and specificity of MRS for detecting tCho in tumours was $78 \%$ and $86 \%$, respectively [25]. Baek et al., measured tCho concentration in a study of 35 breast cancer patients receiving NAC. At first follow-up (after median of 20 days, corresponding with 1-2 cycles of $\mathrm{NAC}$ ), changes in tCho concentrations were not significantly different between patients achieving $\mathrm{pCR}$ and patients not achieving $\mathrm{pCR}$. However, at second follow-up (median of 69 days, corresponding to 3-4 cycles of NAC), tCho concentrations decreased by $100 \%$ in patients achieving pCR (versus $67 \%$ decrease in patients not achieving $p C R, p=0.01$ ) [10].

However, the analysis of the MRS data can be timeconsuming and there are many technical difficulties in assessing the very low concentrations of $\mathrm{tCho}$ in vivo. Other confounding factors are the variable fat content within the breast, field inhomogeneity caused by air-tissue interfaces and marker clips artifacts (inserted after biopsy) [16]. Until now, these limitations prevented a widespread clinical use of this technique to monitor early response in patients receiving NAC.

\section{Other parameters}

In the recent past, a great many parameters have been introduced to monitor early response in the NAC setting. Next to the aforementioned parameters, these include for example early contrast uptake, signal intensity ratio, maximum Gd-DTPA concentration, relative blood volume, relative blood flow, mean transit time, signal intensity time curves, relative signal enhancement after $3,12,17,90$, and $90-450$ seconds, $\mathrm{R}^{*}$ value, intrinsic $\mathrm{T} 2 *$ relaxivity, differences in $\mathrm{R} 2 *$ values, initial area under the gadolinium curve after 60 seconds $\left(\right.$ IAUGC $\left.{ }_{60}\right), B I-R A D S$ lexicon descriptors, change in tumour morphology, early enhanced ratio during the first phase after contrast administration, maximal enhancement ratio, time to maximal enhancement, and peak enhancement ratio [5]. However, these parameters have only been studied in 1 or 2 studies with rather limited population sizes. As a result, there is currently no sufficient evidence that any of these parameters can be used in clinical practice to monitor early response in patients receiving NAC for breast cancer.
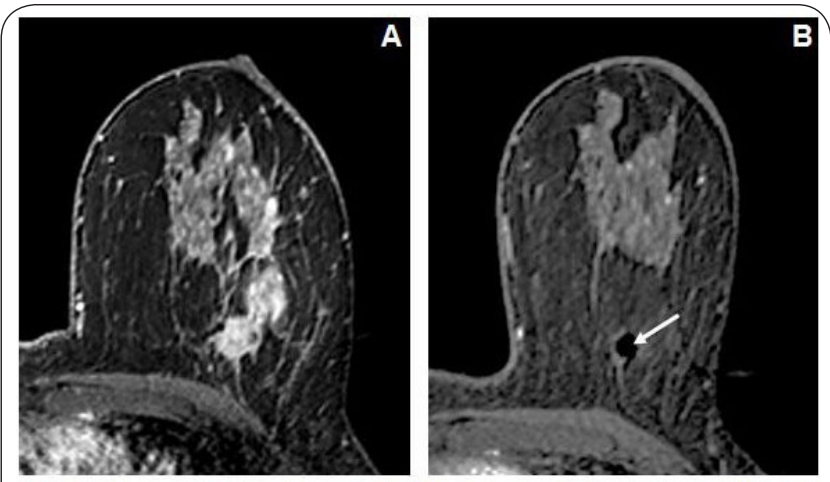

Figure 5. (A) shows a large irregular mass with heterogeneous enhancement in the lateral part of the left breast. After completion of neoadjuvant chemotherapy (B), no residual enhancing tumor is left and only the signal void of the metal marker clip can be observed (arrow). Histopathological analysis of the surgical specimen showed pathologic complete response, i.e. Miller and Payne grade 5.

\section{Residual disease extent and pathologic com- plete response}

After completion of all cycles of NAC, MRI can also be used to assess the extent of residual disease, which can be extremely useful for the surgeon when planning the surgical treatment strategy. It also contains prognostic information, since the achievement of $\mathrm{pCR}$ is associated with better long-term survival. However, the latter can be drawn better from the histopathological analysis of the surgical specimen.

\section{Residual disease extent}

After the completion of NAC, residual disease is still present in the majority of patients. PCR is only achieved by $6-15 \%$, for example in anthracycline-based regimens. PCR rate can increase up to $30 \%$ when taxanes are added [26]. Although several recent studies have focused on the (early) prediction of $\mathrm{pCR}$ by MRI, they failed to provide information about residual disease (which is still present in the majority of patients receiving NAC). Many studies have presented correlation coefficients between size measurements assessed by MRI compared to pathological tumour size measurement $[23,27,42]$. In an analysis of these studies, we calculated that the median correlation coefficient were 0.698 , with a range of 0.21-0.982 (Lobbes, unpublished data). However, most of the studies did not sufficiently discuss the agreement between tumour size measurements as assessed by MRI and histopathology. Excellent correlation coefficients do not automatically reflect excellent agreement.

In summary, the ability of breast MRI to assess residual disease extent seems adequate, but in the various studies on this topic potential confounders for the accuracy of MRI were discovered, which are summarized in the 'Confounding 


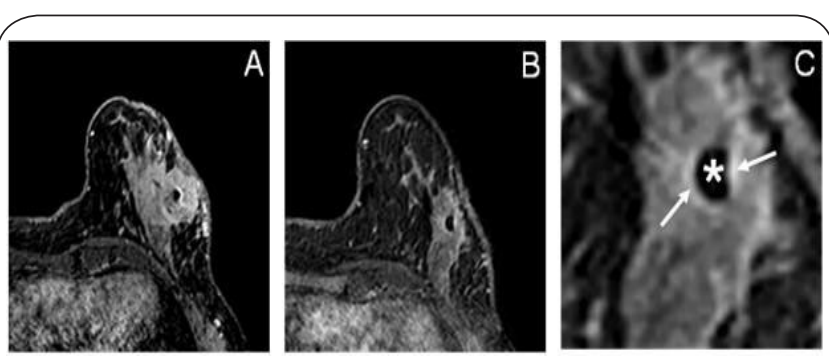

Figure 6. Baseline contrast-enhanced T1-weighted MR image (A), showing a heterogeneously enhancing irregular mass in the left breast with a centrally placed marker clip. Note the adjacent skin thickening. After completion of neoadjuvant chemotherapy (B), the tumour bed reduced slightly, and limited enhancement was observed in this area. Detailed outtake of this area $(\mathbf{C})$ shows the signal void caused by the marker $\left(^{*}\right)$, surrounded by two rims of increased signal intensity (arrows). This might suggest or obscure small foci of residual disease. The configuration of the increased signal intensity parallel to the signal void suggests a susceptibility artifact. The final conclusion of this case was 'probably complete response, but residual disease cannot be ruled out'. Histopathologic analysis showed complete pathologic response (Miller and Payne grade 5).

factors and potential pitfalls' paragraph.

\section{Pathologic complete response (pCR)}

Pathologic complete response is defined as the absence of any invasive tumour cells in the surgical specimen, after the completion of NAC. However, pathological grading systems to assess treatment response vary widely, also in their definition of $\mathrm{pCR}$.

In treatment response monitoring by MRI, the achievement of $p C R$ is suggested by the absence of any residual enhancing tumour tissue after contrast administration (Figure 5). At this stage, histopathological analysis has not yet been performed and therefore we prefer the term 'complete radiological response' in our institution. In a meta-analysis by Yuan et al., the pooled weighted estimates of sensitivity and specificity of breast MRI to predict pCR were 0.63 (range 0.56-0.70) and 0.91 (range 0.91-0.92), respectively [43]. In a meta-analysis of 34 studies by Wu et al., the ability of both DWI and contrastenhanced MRI to predict $\mathrm{pCR}$ was evaluated. They found that sensitivity and specificity for contrast-enhanced MRI was 0.68 (95\% confidence interval (Cl) 0.57-0.77) and 0.91 (95\% $\mathrm{Cl} 0.87-0.94)$, respectively. For DWI, sensitivity and specificity were 0.93 (95\% Cl 0.82-0.97) and 0.82 (95\% Cl 0.70-0.90), respectively [44]. The minor variations in these observations can be explained by the minor differences in inclusion and exclusion criteria that were applied in their reviews.

Based on these critical reviews, it can be concluded that the accuracy of contrast-enhanced MRI to predict PCR after NAC has a high specificity, but only moderate sensitivity. Several confounding factors were observed that might influence its accuracy (see also the 'Confounding factors and potential pitfalls' paragraph). In contrast, the sensitivity of DWI to predict $\mathrm{pCR}$ seems better, albeit at slightly lower specificity. In theory, the combined use of DWI and contrastenhanced MRI could further improve the accuracy of MRI to predict $P C R$, since one MR technique might compensate the shortcomings of the other. However, no studies have been published that have demonstrated the added value of both techniques combined.

\section{Confounding factors and potential pitfalls Marker artifacts}

During the course of NAC, tumour might regress in diameter and become more difficult to visualize by imaging. In the best case scenario, complete response is observed and the tumour is not detectable at all anymore. Therefore, all patients receiving NAC and in which breast-conserving therapy might be considered afterwards should have their tumour marked. In general, this is done by placing a clip, marker or small coil inside the tumour. However, these clips cause local field inhomogeneities when MRI is performed, causing susceptibility artifacts in the tumour bed itself. These artifacts are usually observed as a signal void (which represents the marker itself), surrounded by a rim of increased signal intensity, which is often more pronounced than the enhancing fibroglandular tissue or residual disease. However, these small areas of increased signal intensity might be difficult to distinguish from small foci of residual disease (Figure 6). Subtraction of contrastenhanced MR images might aid in reducing these false positive findings.

Breast cancer subtypes and chemotherapy regimen In recent studies, it was observed that the accuracy of breast MRI to assess residual disease and predict pCR depended on breast cancer subtypes, but also on the chemotherapy regimen that was used. For example, it was observed that MRI was able to predict $\mathrm{pCR}$ more accurately in patients having HER2-positive tumours when compared to HER2-negative tumours. In the latter case, a higher falsenegative rate was observed, especially when receiving anti-angiogenic drugs $[\mathbf{4 0 , 4 5 ]}$. It was also observed that the average size discrepancy between MRI and pathology was greater in tumours demonstrating limited Ki- 67 staining when compared to tumours demonstrating increased $\mathrm{Ki}-67$ staining [40]. In addition, Loo et al., concluded that response monitoring using breast $\mathrm{MRI}$ is accurate in patients having triple-negative or HER2-positive tumours. However, they found it was inaccurate in ER-positive/HER2negative subtypes [46]. Finally, Denis et al., showed that residual tumour size was often underestimated by MRI in taxane-containing regimens, which is probably caused by the antivascular effects of this type of chemotherapy, which results in less enhancement on contrast-enhanced breast MRI [47]. These findings suggest that breast cancer subtype and the type of NAC is of influence on breast MRI 
accuracy. Therefore, larger studies are warranted to further clarify which factors effect MRI's accuracy and in what way (positive or negative).

\section{Overestimation and underestimation}

In monitoring response to NAC by MRI, both overestimation and underestimation were observed in various studies. Both discrepancies can have an important impact on patient care. Overestimation of tumour size by MRI can be caused by surrounding sclerosis or necrosis, the presence of multiple scattered lesions or foci, reactive inflammation caused by tumour response and subsequent healing, and the presence of accompanying ductal carcinoma in situ $[31,48,49]$. If the residual tumour size is overestimated, it could alter the surgical treatment plan for the individual patient. It could result in unnecessary wide resection margins with subsequent poorer cosmetic results, but it could also result in a mastectomy where breast-conserving therapy might still have been possible.

Underestimation of residual tumour size by MRI can be caused by the lack of an inflammatory response surrounding the tumour (common in regimens containing docetaxel), the antivascular effects of docetaxel, extensive ductal carcinoma in situ component (which may be difficult to identify by $\mathrm{MRI}$ ), and partial volume effects in the case of very small foci of residual disease $[\mathbf{2 7}, \mathbf{3 0}, \mathbf{4 7}]$. The risk of positive resection margins after surgery increases in these cases. Studies have shown that positive resection margins are associated with increased risk of disease recurrence [50]. In addition, positive resection margins require additional re-excisions or even conversion to mastectomy. This increases health care costs due to additional surgeries that need to performed, and the additional hospital admission times that are required in these cases.

\section{Conclusions and future perspectives}

Currently, breast MRI is considered the most accurate method for evaluating treatment response in breast cancer patients receiving NAC. Even after several cycles of NAC, changes in tumour size, morphology, enhancement and metabolism can be observed in vivo. Based on these changes, a great many parameters have been proposed for early response monitoring of NAC by MRI. Of these parameters, tumour size and volume, and ADC values seem most promising and are best validated. However, proper response criteria (such as optimal cut off values) are still lacking for these parameters and should be investigated in future (larger) studies.

It might be assumed that early response monitoring could be used to change treatment strategy during NAC. In theory, patients that showed a poor response to one chemotherapy regimen might switch to an alternative one. However, several large randomized clinical trials did not support this hypothesis [5]. Early response monitoring might be used for the distinction between good and partial responders. When good response is observed, the selected regimen could be continued as planned. Patients that showed only partial response might benefit from a more prolonged chemotherapy treatment (i.e. more cycles of chemotherapy), in order to achieve a higher pCR rate. In non-responders, or even progressive disease, one might reconsider surgical intervention at an earlier stage in treatment or at least prevent the administration of toxic agents from which the patient does not benefit. However, the application of breast MRI for early response monitoring is still developing at this moment, and so far, no studies have been published that altered their treatment plan based on the breast MRI findings.

The accuracy of breast MRI to predict pathologic complete response has a moderate sensitivity, but high specificity. DWI might be able to compensate this shortcoming, since it has higher sensitivity and only slightly lower specificity. Unfortunately, there are no studies yet that have investigated the added value of both MR techniques combined.

However, pCR only occurs in the minority of patients. Residual disease assessment after NAC by MRI might be even more important. Studies have shown that assessment of residual disease by MRI is good, but can be influenced by several factors, such as breast cancer subtype or treatment regimen. Finally, both overestimation and underestimation of residual disease by breast MRI have been reported in the past. Both can have important clinical impact for the individual patient.

Future studies should further investigate the confounding variables that could influence the accuracy of breast MRI during NAC. Researchers should not only focus on single MR techniques, but rather explore the added value of combined MR techniques that might compensate for the separate shortcomings of the various techniques. However, study designs for these studies should consist of large, multicenter trials, since most available studies only included a limited number of patients. Underpowered studies could mask important findings which would be statistically significant if the population size was adequately powered.

\section{Competing interests}

The authors declare that they have no competing interests.

\section{Author contributions}

$M L, R P$, and MS all contributed to this manuscript. Manuscript preparation was performed by ML and MS. Figure preparation was performed by RP. ML and RP provided the unpublished data presented in this manuscript. All authors read and approved the final manuscript.

Publication history

Received: 17-Nov-2012 Revised: 08-Dec-2012

Accepted: 12-Dec-2012 Published: 19-Dec-2012

\section{References}

1. D. Mauri, N. Pavlidis and J. P. loannidis: Neoadjuvant versus adjuvant systemic treatment in breast cancer: a meta-analysis. J Nat/ Cancer Inst 2005, 97:188-94. | Article | PubMed

2. X. Kong, M. S. Moran, N. Zhang, B. Haffty and Q. Yang: Metaanalysis confirms achieving pathological complete response after 
neoadjuvant chemotherapy predicts favourable prognosis for breast cancer patients. Eur J Canc 2011, 47:2084-90. | Article | PubMed

3. A. B. Chagpar, L. P. Middleton, A. A. Sahin, P. Dempsey, A. U. Buzdar, A. N. Mirza, F. C. Ames, G. V. Babiera, B. W. Feig, K. K. Hunt, H. M. Kuerer, F. Meric-Bernstam, M. I. Ross and S. E. Singletary: Accuracy of physical examination, ultrasonography, and mammography in predicting residual pathologic tumor size in patients treated with neoadjuvant chemotherapy. Ann Surg 2006, 243:257-64. | Article | PubMed Abstract | PubMed Full Text

4. N. M. Hylton, J. D. Blume, W. K. Bernreuter, E. D. Pisano, M. A. Rosen, E. A. Morris, P. T. Weatherall, C. D. Lehman, G. M. Newstead, S. Polin, H. S. Marques, L. J. Esserman and M. D. Schnall: Locally advanced breast cancer: MR imaging for prediction of response to neoadjuvant chemotherapy--results from ACRIN 6657/I-SPY TRIAL. Radiology 2012, 263:663-72. | Article | PubMed

5. R. Prevos, M. L. Smidt, V. C. Tjan-Heijnen, M. van Goethem, R. G. Beets-Tan, J. E. Wildberger and M. B. Lobbes: Pre-treatment differences and early response monitoring of neoadjuvant chemotherapy in breast cancer patients using magnetic resonance imaging: a systematic review. Eur Radiol 2012, 22:2607-16. | Article I PubMed

6. J. P. O'Connor, A. Jackson, G. J. Parker and G. C. Jayson: DCE-MRI biomarkers in the clinical evaluation of antiangiogenic and vascular disrupting agents. Br J Cancer 2007, 96:189-95. | Article | PubMed Abstract | PubMed Full Text

7. S. Zwick, G. Brix, P. S. Tofts, R. Strecker, A. Kopp-Schneider, H. Laue, W. Semmler and F. Kiessling: Simulation-based comparison of two approaches frequently used for dynamic contrast-enhanced MRI. Eur Radiol 2010, 20:432-42. | Article | PubMed

8. H. J. Yu, J. H. Chen, R. S. Mehta, O. Nalcioglu and M. Y. Su: MRI measurements of tumor size and pharmacokinetic parameters as early predictors of response in breast cancer patients undergoing neoadjuvant anthracycline chemotherapy. JMRI 2007, 26:615-23. | Article I PubMed

9. M. L. Ah-See, A. Makris, N. J. Taylor, M. Harrison, P. I. Richman, R. J. Burcombe, J. J. Stirling, J. A. d'Arcy, D. J. Collins, M. R. Pittam, D. Ravichandran and A. R. Padhani: Early changes in functional dynamic magnetic resonance imaging predict for pathologic response to neoadjuvant chemotherapy in primary breast cancer. Clin Cancer Res 2008, 14:6580-9. | Article | PubMed

10. H. M. Baek, J. H. Chen, K. Nie, H. J. Yu, S. Bahri, R. S. Mehta, O. Nalcioglu and M. Y. Su: Predicting pathologic response to neoadjuvant chemotherapy in breast cancer by using MR imaging and quantitative 1H MR spectroscopy. Radiology 2009, 251:653-62. I Article | PubMed Abstract | PubMed Full Text

11. S. P. Li, N. J. Taylor, A. Makris, M. L. Ah-See, M. J. Beresford, J. J. Stirling, J. A. d'Arcy, D. J. Collins and A. R. Padhani: Primary human breast adenocarcinoma: imaging and histologic correlates of intrinsic susceptibility-weighted MR imaging before and during chemotherapy. Radiology 2010, 257:643-52. | Article | PubMed

12. Y. Yu, Q. Jiang, Y. Miao, J. Li, S. Bao, H. Wang, C. Wu, X. Wang, J. Zhu, Y. Zhong, E. M. Haacke and J. Hu: Quantitative analysis of clinical dynamic contrast-enhanced MR imaging for evaluating treatment response in human breast cancer. Radiology 2010, 257:47-55. | Article | PubMed Abstract | PubMed Full Text

13. C. Kuhl: The current status of breast MR imaging. Part I. Choice of technique, image interpretation, diagnostic accuracy, and transfer to clinical practice. Radiology 2007, 244:356-78. | Article | PubMed

14. C. E. Loo, H. J. Teertstra, S. Rodenhuis, M. J. van de Vijver, J. Hannemann, S. H. Muller, M. J. Peeters and K. G. Gilhuijs: Dynamic contrast-enhanced MRI for prediction of breast cancer response to neoadjuvant chemotherapy: initial results. AJR Am J Roentgenol 2008, 191:1331-8. | Article | PubMed

15. A. B. Miller, B. Hoogstraten, M. Staquet and A. Winkler: Reporting results of cancer treatment. Cancer 1981, 47:207-14. | Article | PubMed

16. R. McLaughlin and N. Hylton: MRI in breast cancer therapy monitoring. NMR Biomed 2011, 24:712-20. | Article | PubMed
17. P. Therasse, S. G. Arbuck, E. A. Eisenhauer, J. Wanders, R. S. Kaplan, L. Rubinstein, J. Verweij, M. Van Glabbeke, A. T. van Oosterom, M. C. Christian and S. G. Gwyther: New guidelines to evaluate the response to treatment in solid tumors. European Organization for Research and Treatment of Cancer, National Cancer Institute of the United States, National Cancer Institute of Canada. J Natl Cancer Inst 2000, 92:205-16. | Article | PubMed

18. M. Van Goethem, K. Schelfout, E. Kersschot, C. Colpaert, I. Verslegers, I. Biltjes, W. A. Tjalma, A. De Schepper, J. Weyler and P. M. Parizel: MR mammography is useful in the preoperative locoregional staging of breast carcinomas with extensive intraductal component. Eur J Radiol 2007, 62:273-82. | Article | PubMed

19. M. Lorenzon, C. Zuiani, V. Londero, A. Linda, A. Furlan and M. Bazzocchi: Assessment of breast cancer response to neoadjuvant chemotherapy: is volumetric MRI a reliable tool? Eur J Radiol 2009, 71:82-8. | Article | PubMed

20. P. C. Tan, M. D. Pickles, M. Lowry, D. J. Manton and L. W. Turnbull: Lesion T(2) relaxation times and volumes predict the response of malignant breast lesions to neoadjuvant chemotherapy. Magn Reson Imaging 2008, 26:26-34. | Article | PubMed

21. M. D. Pickles, M. Lowry, D. J. Manton, P. Gibbs and L. W. Turnbull: Role of dynamic contrast enhanced MRI in monitoring early response of locally advanced breast cancer to neoadjuvant chemotherapy. Breast Cancer Res Treat 2005, 91:1-10. | Article | PubMed

22. U. Sharma, K. K. Danishad, V. Seenu and N. R. Jagannathan: Longitudinal study of the assessment by MRI and diffusion-weighted imaging of tumor response in patients with locally advanced breast cancer undergoing neoadjuvant chemotherapy. ccc 2009, 22:104-13. | Article | PubMed

23. A. Fangberget, L. B. Nilsen, K. H. Hole, M. M. Holmen, O. Engebraaten, B. Naume, H. J. Smith, D. R. Olsen and T. Seierstad: Neoadjuvant chemotherapy in breast cancer-response evaluation and prediction of response to treatment using dynamic contrast-enhanced and diffusion-weighted MR imaging. Eur Radiol 2011, 21:1188-99. | Article | PubMed Abstract | PubMed Full Text

24. X. R. Li, L. Q. Cheng, M. Liu, Y. J. Zhang, J. D. Wang, A. L. Zhang, X. Song, J. Li, Y. Q. Zheng and L. Liu: DW-MRI ADC values can predict treatment response in patients with locally advanced breast cancer undergoing neoadjuvant chemotherapy. Med Oncol 2012, 29:425-31. | Article | PubMed

25. N. R. Jagannathan, M. Kumar, V. Seenu, O. Coshic, S. N. Dwivedi, P. K. Julka, A. Srivastava and G. K. Rath: Evaluation of total choline from in-vivo volume localized proton MR spectroscopy and its response to neoadjuvant chemotherapy in locally advanced breast cancer. $\mathrm{Br}$ J Cancer 2001, 84:1016-22. | Article | PubMed Abstract | PubMed Full Text

26. A. M. Gonzalez-Angulo, F. Morales-Vasquez and G. N. Hortobagyi: Overview of resistance to systemic therapy in patients with breast cancer. Adv Exp Med Biol 2007, 608:1-22. | Article | PubMed

27. S. C. Partridge, J. E. Gibbs, Y. Lu, L. J. Esserman, D. Sudilovsky and N. $M$. Hylton: Accuracy of MR imaging for revealing residual breast cancer in patients who have undergone neoadjuvant chemotherapy. AJR Am J Roentgenol 2002, 179:1193-9. | PubMed

28. Y. C. Cheung, S. C. Chen, M. Y. Su, L. C. See, S. Hsueh, H. K. Chang, Y. C. Lin and C. S. Tsai: Monitoring the size and response of locally advanced breast cancers to neoadjuvant chemotherapy (weekly paclitaxel and epirubicin) with serial enhanced MRI. Breast Canc Res Treat 2003, 78:51-8. | Article | PubMed

29. R. M. Warren, L. G. Bobrow, H. M. Earl, P. D. Britton, D. Gopalan, A. D. Purushotham, G. C. Wishart, J. R. Benson and W. Hollingworth: Can breast MRI help in the management of women with breast cancer treated by neoadjuvant chemotherapy? Br J Canc 2004, 90:1349-60. | Article | PubMed Abstract | PubMed Full Text

30. D. Segara, I. E. Krop, J. E. Garber, E. Winer, L. Harris, J. R. Bellon, R. Birdwell, S. Lester, S. Lipsitz, J. D. Iglehart and M. Golshan: Does MRI predict pathologic tumor response in women with breast cancer undergoing preoperative chemotherapy? J Surg Oncol 2007, 96:47480. | Article | PubMed

31. H. J. Kim, Y. H. Im, B. K. Han, N. Choi, J. Lee, J. H. Kim, Y. L. Choi, J. S. 
Ahn, S. J. Nam, Y. S. Park, Y. H. Choe, Y. H. Ko and J. H. Yang: Accuracy of MRI for estimating residual tumor size after neoadjuvant chemotherapy in locally advanced breast cancer: relation to response patterns on MRI. Acta Oncologica 2007, 46:996-1003. I Article I PubMed

32. H. G. Moon, W. Han, J. W. Lee, E. Ko, E. K. Kim, J. H. Yu, S. Y. Kang, W. K. Moon, N. Cho, I. A. Park, D. Y. Oh, S. W. Han, S. A. Im and D. Y. Noh: Age and HER2 expression status affect MRI accuracy in predicting residual tumor extent after neo-adjuvant systemic treatment. Ann Oncol 2009, 20:636-41. | Article | PubMed

33. F. C. Wright, J. Zubovits, S. Gardner, B. Fitzgerald, M. Clemons, M. L. Quan and P. Causer: Optimal assessment of residual disease after neo-adjuvant therapy for locally advanced and inflammatory breast cancer--clinical examination, mammography, or magnetic resonance imaging? J Surg Oncol 2010, 101:604-10. | Article | PubMed

34. S. H. Park, W. K. Moon, N. Cho, I. C. Song, J. M. Chang, I. A. Park, W. Han and D. Y. Noh: Diffusion-weighted MR imaging: pretreatment prediction of response to neoadjuvant chemotherapy in patients with breast cancer. Radiology 2010, 257:56-63. | Article | PubMed

35. H. Nakahara, Y. Yasuda, E. Machida, Y. Maeda, H. Furusawa, K. Komaki, M. Funagayama, M. Nakahara, S. Tamura and F. Akiyama: MR and US imaging for breast cancer patients who underwent conservation surgery after neoadjuvant chemotherapy: comparison of triple negative breast cancer and other intrinsic subtypes. Breast Cancer 2011, 18:152-60. | Article | PubMed

36. Wang X, Peng W, Tan H, Xin C, Mao J: Evaluation of dynamic contrastenhanced MRI in monitoring early response of locally advanced breast cancer to neoadjuvant chemotherapy. Chinese-German J Clin Oncol 2010, 9:637-42. | Article

37. H. Dongfeng, M. Daqing and J. Erhu: Dynamic breast magnetic resonance imaging: pretreatment prediction of tumor response to neoadjuvant chemotherapy. Clinical Breast Cancer 2012, 12:94-101. | Article | PubMed

38. V. Guarneri, A. Pecchi, F. Piacentini, E. Barbieri, M. V. Dieci, G. Ficarra, G. Tazzioli, A. Frassoldati, R. Battista, B. Canossi, C. Mauri, R. D'Amico, P. Conte and P. Torricelli: Magnetic resonance imaging and ultrasonography in predicting infiltrating residual disease after preoperative chemotherapy in stage II-III breast cancer. Ann Surg Oncol 2011, 18:2150-7. | Article | PubMed

39. H. J. Shin, H. H. Kim, J. H. Ahn, S. B. Kim, K. H. Jung, G. Gong, B. H. Son and S. H. Ahn: Comparison of mammography, sonography, MRI and clinical examination in patients with locally advanced or inflammatory breast cancer who underwent neoadjuvant chemotherapy. Br J Radiol 2011, 84:612-20. | $\underline{\text { Article | PubMed }}$ Abstract I PubMed Full Text

40. J. H. Chen, S. Bahri, R. S. Mehta, A. Kuzucan, H. J. Yu, P. M. Carpenter, S. A. Feig, M. Lin, D. J. Hsiang, K. T. Lane, J. A. Butler, O. Nalcioglu and M. Y. Su: Breast cancer: evaluation of response to neoadjuvant chemotherapy with 3.0-T MR imaging. Radiology 2011, 261:735-43. | Article | PubMed Abstract | PubMed Full Text

41. T. H. Kim, D. K. Kang, H. Yim, Y. S. Jung, K. S. Kim and S. Y. Kang: Magnetic resonance imaging patterns of tumor regression after neoadjuvant chemotherapy in breast cancer patients: correlation with pathological response grading system based on tumor cellularity. J Comput Assist Tomogr 2012, 36:200-6. | Article | PubMed

42. H. J. Shin, H. M. Baek, J. H. Ahn, S. Baek, H. Kim, J. H. Cha and H. H. Kim: Prediction of pathologic response to neoadjuvant chemotherapy in patients with breast cancer using diffusionweighted imaging and MRS. NMR Biomed 2012, 25:1349-59. | Article I PubMed

43. Y. Yuan, X. S. Chen, S. Y. Liu and K. W. Shen: Accuracy of MRI in prediction of pathologic complete remission in breast cancer after preoperative therapy: a meta-analysis. AJR Am J Roentgenol 2010, 195:260-8. | Article | PubMed

44. L. M. Wu, J. N. Hu, H. Y. Gu, J. Hua, J. Chen and J. R. Xu: Can diffusionweighted MR imaging and contrast-enhanced MR imaging precisely evaluate and predict pathological response to neoadjuvant chemotherapy in patients with breast cancer? Breast Cancer Res Treat 2012, 135:17-28. | Article | PubMed

45. J. H. Chen, B. Feig, G. Agrawal, H. Yu, P. M. Carpenter, R. S. Mehta, O. Nalcioglu and M. Y. Su: MRI evaluation of pathologically complete response and residual tumors in breast cancer after neoadjuvant chemotherapy. Cancer 2008, 112:17-26. | Article | PubMed

46. C. E. Loo, M. E. Straver, S. Rodenhuis, S. H. Muller, J. Wesseling, M. J. Vrancken Peeters and K. G. Gilhuijs: Magnetic resonance imaging response monitoring of breast cancer during neoadjuvant chemotherapy: relevance of breast cancer subtype. J Clin Oncol 2011, 29:660-6. | Article | PubMed

47. F. Denis, A. V. Desbiez-Bourcier, C. Chapiron, F. Arbion, G. Body and L. Brunereau: Contrast enhanced magnetic resonance imaging underestimates residual disease following neoadjuvant docetaxel based chemotherapy for breast cancer. EJSO 2004, 30:1069-76. | Article I PubMed

48. E. Yeh, P. Slanetz, D. B. Kopans, E. Rafferty, D. Georgian-Smith, L. Moy, E. Halpern, R. Moore, I. Kuter and A. Taghian: Prospective comparison of mammography, sonography, and MRI in patients undergoing neoadjuvant chemotherapy for palpable breast cancer. AJR Am J Roentgenol 2005, 184:868-77. | PubMed

49. P. Belli, M. Costantini, C. Malaspina, A. Magistrelli, G. Latorre and L. Bonomo: MRI accuracy in residual disease evaluation in breast cancer patients treated with neoadjuvant chemotherapy. Clin Radiol 2006, 61:946-53. | Article | PubMed

50. D. Cowen, G. Houvenaeghel, V. Bardou, J. Jacquemier, E. Bautrant, M. Conte, P. Viens, R. Largillier, B. Puig, M. Resbeut and D. Maraninchi: Local and distant failures after limited surgery with positive margins and radiotherapy for node-negative breast cancer. Int J Radiat Oncol Biol Phys 2000, 47:305-12. | Article | PubMed

\section{Citation:}

Lobbes M, Prevos R and Smidt M: Response monitoring of breast cancer patientsreceiving neoadjuvant chemotherapy using breast MRI a review of current knowledge. journal of Cancer Therapeutics and Research 2012, 1:34. http://dx.doi.org/10.7243/2049-7962-1-34 